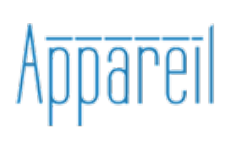

Appareil

$23 \mid 2021$

Poïétique du jeu vidéo

\title{
Théâtre immersif et jeu vidéo
}

Éléments d'analyse sociocritique du processus de création participative

\section{Olivia Levet}

\section{(2) OpenEdition}

Journals

Édition électronique

URL : http://journals.openedition.org/appareil/3991

DOI : 10.4000/appareil.3991

ISSN : 2101-0714

Éditeur

MSH Paris Nord

Référence électronique

Olivia Levet, « Théâtre immersif et jeu vidéo », Appareil [En ligne], 23 | 2021, mis en ligne le 31 mars 2021, consulté le 02 avril 2021. URL : http://journals.openedition.org/appareil/3991 ; DOI : https:// doi.org/10.4000/appareil.3991

Ce document a été généré automatiquement le 2 avril 2021.

\section{(c)}

Appareil est mis à disposition selon les termes de la Licence Creative Commons Attribution - Pas d'Utilisation Commerciale - Pas de Modification 4.0 International. 


\title{
Théâtre immersif et jeu vidéo
}

\author{
Éléments d'analyse sociocritique du processus de création participative
}

\section{Olivia Levet}

\section{Introduction}

1 Brenda Laurel, en 1991, propose dans son ouvrage Computers as Theatre ${ }^{1}$ un premier rapprochement entre le jeu vidéo et le théâtre, au regard de l'interaction et du design d'interface. Elle s'appuie sur La Poétique d'Aristote, afin de penser l'expérience utilisateur à partir de la mimêsis. L'interface numérique devient une scène de théâtre, une représentation du monde au sein de laquelle l'utilisateur va pouvoir jouer un rôle à la première personne, manipuler un univers naturel, cohérent, convivial et familier. À l'heure où l'immersion est un argument courant dans le domaine du marketing et un but à atteindre, à la fois dans le domaine du jeu vidéo et dans celui du théâtre avec l'apparition d'un théâtre immersif, le lien entre les deux pratiques, au prisme de l'expérience utilisateur (user experience, UX), est central et ambivalent. La prise en compte du public est envisagée sous le règne de la participation, omniprésente au sein d'un système néo-libéral, mais peut également être considérée comme une étape vers une émancipation et une «libération » du public - et de l'auteur - face aux idéologies dominantes. À la suite de l'analyse croisée d'œuvres de théâtre immersif et de procédés de game design (conception de jeux), cet article observera le rapprochement entre résidence et playtest afin d'étudier les enjeux de la prise en compte du public dans la création, qu'elle soit théâtrale ou vidéoludique.

2 Là où le théâtre et les arts vivants influencent le jeu vidéo et le design d'interface, dans la compréhension de l'interaction homme-machine, l'impact culturel du jeu vidéo au sein de notre société contemporaine et technologique devient une inspiration pour renouveler les pratiques théatrales à l'heure du participatif et de l'interactif, tant dans l'élaboration de ces dernières que dans leur conception esthétique. 


\section{La résidence théâtrale comme expérience utilisateur néolibérale}

\section{Greenhive Green de Punchdrunk Enrichment}

Dans son ouvrage Immersive Theatres ${ }^{2}$, Josephine Machon note comment les expériences théâtrales interdisciplinaires plaçant le public au cœur de l'expérience, ou théâtre immersif, se multiplient depuis quelques années. Elles investissent des lieux divers, publics ou privés, afin de proposer des expériences interactives et participatives plongeant les spectateurs dans des bains de sensations fictionnels. Le théâtre immersif apparaît dans les pays anglo-saxons dans les années 2000, notamment avec la compagnie Punchdrunk créée par Felix Barett à Londres. En 2008 naît Punchdrunk Enrichment, sous la direction de Peter Higgin, afin de travailler plus particulièrement avec des communautés ou des écoles, dans une démarche éducative et sociale.

En 2016, Punchdrunk Enrichment travaille huit semaines en résidence au sein de la maison de soins (care home) Greenhive à Peckham, donnant lieu à l'œuvre Greenhive Green. Ici, la résidence constitue l'œuvre elle-même; elle s'inscrit dans un programme réunissant artistes, personnel soignant et personnes âgées, mis en place par l'association caritative Magic $\mathrm{Me}^{3}$, en partenariat avec Anchor Hanover ${ }^{4}$, dans des maisons de soins. L'enjeu, ici, est de s'adapter à la structure d'accueil et à son public atteint de démences et troubles cognitifs, tout en gardant la spécificité artistique de Punchdrunk, comme spécifié dans le rapport de Magic Me de 2017, « Magic me’s artists residencies in care homes »:

Les quatre partenaires artistiques étaient tous prêts à relever le défi de travailler avec et pour ce public très différent, dans un environnement très particulier, tout en préservant l'intégrité de leur propre pratique et leur esthétique unique ${ }^{5}$.

5 Les résidents sont invités à participer chaque mardi aux activités quotidiennes du village fictif de Greenhive Green, dont l'histoire va évoluer au fur et à mesure de la résidence. Ils incarnent le Comité du village, organe décisionnaire dans les relations avec le village voisin, Blarford ${ }^{6}$. La démarche de la compagnie est de sortir les résidents de leur quotidien en s'adaptant à leurs capacités physiques et mentales, mais aussi de créer un fort sentiment de communauté.

[...] l'espace est totalement transformé visuellement, avec du son spatialisé, des odeurs, des textures et des lumières. L'ambition était de créer un bel espace pour les résidents, améliorant leur environnement et mettant en place une activité intéressante dans le foyer, mais aussi de les transporter dans un environnement différent issu de notre univers ${ }^{7}$.

Le projet est décrit dans le rapport de Magic Me comme un mélange de jeu et de feuilleton ${ }^{8}$. Le format de la résidence permet d'expérimenter avec les habitants et de faire évoluer la pièce et la fiction en fonction de leurs propositions, envies et implications, en cohérence avec leurs capacités.

Le récit prévu sur l'histoire de deux villages a été simplifié peu de temps après le début de la résidence de 8 semaines, quand nous avons réalisé qu'il était inutile et difficile pour beaucoup de résidents de suivre le fil de semaine en semaine. L'histoire s'est alors centrée sur deux villages voisins devenant amis à travers des échanges - de l'envoi des Gâteaux de la Diplomatie au choix de l'hymne du village'.

Cette œuvre diffère du travail mené habituellement par Punchdrunk : la fiction prend place dans le lieu de vie des participants, en s'appuyant sur leurs habitudes, et s'étend 
sur une temporalité plus longue. L'accent est mis sur leurs échanges et discussions et non sur une déambulation libre du public dans l'espace de représentation, comme cela est habituellement le cas dans les pièces de la compagnie ${ }^{10}$. Certaines caractéristiques perdurent tout de même : mise en place d'un univers brouillant les limites entre monde physique et virtuel, fort accent mis sur l'aspect magique, mystérieux et viscéral et création d'une narration principalement centrée sur le spectateur: " Dans sa forme la plus simple, le projet était un lieu agréable dans lequel nous pouvions nous rencontrer chaque semaine pour partager une tasse de thé et faire une activité enrichissante. D'un point de vue plus complexe, c'était un lieu où se déployait une narration, une narration dont [les participants] sont (sic) au centre et dont ils sont les protagonistes, un monde de mystère et de détail ${ }^{11}$. " Par ailleurs, la résidence, derrière une volonté affichée d'améliorer les conditions de vie au sein de la maison, ne remet jamais en question le manque de moyens économiques de ces établissements et ne s'aventure aucunement sur un terrain politique, préférant proposer des activités manuelles et divertissantes aux résidents.

7 Cette pièce, comme l'ensemble des œuvres de "théâtre immersif », renoue avec un théâtre souhaitant briser les conventions classiques et expérimentant autour de la relation avec le public. Ces œuvres ont également des affinités affichées avec le domaine du jeu: elles rappellent en effet les expériences de jeu de rôle grandeur nature, où la trame constitue une base à l'improvisation des joueurs et à l'incarnation de personnages. Felix Barett revendique l'influence de jeux vidéo d'aventure à la première personne ${ }^{12}$ tels que Gone $H_{0} e^{13}$ dans l'élaboration de la pièce The Drowned $\mathrm{Man}^{14}$. Les jeux vidéo en 3D proposant l'exploration d'une "narration environnementale » reposent en effet sur des mécaniques et outils similaires: ils affirment de nouvelles modalités narratives en proposant à l'utilisateur de jouer un rôle et d'interpréter une narration via la traversée d'un environnement, d'un monde cohérent, constitué de scènes. Henry Jenkins, en théorisant le jeu vidéo comme architecture narrative actualisée par la participation du joueur, affirme déjà cet aspect comme un possible héritage de la Commedia dell'arte: le metteur en scène définit les règles et attributs propres aux personnages joués via les masques portés par les acteurs. Ces derniers interprètent alors ce cadre narratif par l'improvisation, à la manière des joueurs de jeux vidéo ${ }^{15}$.

8 Au-delà du vocabulaire et des procédés communs aux deux domaines, Greenhive Green illustre parfaitement un processus de gamification ${ }^{16}$ : le jeu vidéo s'insinue dans l'ensemble des aspects de notre quotidien afin de "réenchanter le monde ${ }^{17}$ » (Siegel, 2015). Le théâtre immersif, en plaçant les spectateurs dans la position de héros de jeux vidéo " magiques » et spectaculaires, s'adapte à une société où le ludique s'impose dans un ensemble de domaines et dicte les attentes du public vers un art de plus en plus immersif et interactif.

\section{Expérience utilisateur et game design}

9 La définition de l'immersion renvoie au fait de plonger dans un liquide, de passer d'un état, d'un environnement à l'autre. Lorsque Josephine Machon définit ce concept, interchangeable selon les auteurs et champs d'étude avec le concept de "presence " (présence), elle note son imprécision. Affirmant l'existence de spécificités communes entre le théâtre et le jeu vidéo, l'autrice s'appuie sur les game studies et notamment sur 
l'ouvrage In-game: from immersion to incorporation ${ }^{18}$ de Gordon Calleja, afin de comprendre les spécificités du concept. En appliquant l'analyse de ce dernier au domaine du théâtre, Josephine Machon identifie le théâtre immersif comme immersion totale réunissant à la fois l'«immersion comme absorption » et l'« immersion comme transport». Elle souligne néanmoins une différence entre le théâtre immersif, où l'environnement est imaginaire et sensoriel, mais aussi physique, et le jeu vidéo.

10 Il est intéressant de noter que l'autrice puise dans les game studies pour penser l'immersion spécifique au théâtre immersif, quand des théoriciennes telles que Brenda Laurel, conceptrice et chercheuse dans le domaine du jeu vidéo et du design d'interface, se sont précédemment appuyées sur le théâtre pour analyser les interfaces numériques et développer des univers conviviaux, fluides et naturels pour les utilisateurs. Dans le domaine des jeux vidéo industriels, l'immersion est aujourd'hui un objectif à atteindre pour beaucoup de concepteurs ; le jeu vidéo doit être le plus réaliste et cohérent possible, projetant les joueurs au sein d'univers vertigineux, où les notions de temps et d'espace sont diluées. La réflexion à propos de l'interface utilisateur (user interface) et l'expérience utilisateur (user experience) permettent ainsi de prendre en compte le joueur dans l'élaboration d'un système répondant de manière cohérente à ses actions. La mise en place de playtests réguliers, visant à tester la jouabilité, l'ergonomie et la convivialité d'un gameplay ${ }^{19}$ afin d'anticiper et d'éviter toute frustration du joueur, trouve alors des échos dans l'organisation de résidences théâtrales participatives, grâce auxquelles les compagnies théâtrales réfléchissent, expérimentent et répètent plus ou moins publiquement, mettant ainsi leur création à l'épreuve d'un premier public.

11 Lors d'un entretien ${ }^{20}$ réalisé pour les besoins de cet article, Michael Freudenthal et Mélanie Dorey, créatrices ${ }^{21}$ de la pièce The Lost Generation, sont revenues sur la manière dont les outils pratiques et théoriques issus du domaine du jeu ont influencé le développement de la pièce, aussi bien dans l'écriture et la mise en scène que dans la manière d'appréhender le rapport au public.

Mélanie Dorey, artiste issue des domaines du cinéma d'animation et de l'art contemporain, crée la compagnie Madame Lupin en 2015. En 2019 naît l'idée de The Lost Generation, une " fête immersive ${ }^{22}$ » sur la période de l'entre-deux-guerres et ce que l'on nomme la «Génération perdue ». Michael Freudenthal, issu des domaines de la communication, de la gestion de projet web mais également du jeu vidéo et du jeu de rôle grandeur nature ${ }^{23}$, rejoint Mélanie Dorey sur le travail de la pièce en tant que play designer ${ }^{24}$.

Dans l'élaboration de The Lost Generation, les conceptrices ont porté une attention particulière aux feedbacks (retours d'information), c'est-à-dire, ici, à la manière dont les acteurs vont répondre aux actions des spectateurs par l'improvisation, afin de les impliquer dans la narration lors de la "représentation » : "Toute implication dans la fiction doit être valorisée ${ }^{25} »$. Deux répétitions générales ouvertes au public, nommées " playtests », ont alors permis de revenir sur l'expérience de chaque participant et ainsi d'améliorer l'engagement du public au sein de la fiction. Le but était d'arriver à un effacement du système, à un passage fluide et invisible entre les moments improvisés et les moments mis en scène, proche des interfaces seamless ${ }^{26}$ de jeux vidéo. Au cours de leur processus créatif, les théories propres à l'UX pour penser l'« onboarding ${ }^{27}$ » des spectateurs dans l'univers de la pièce, mais également les recherches liées aux game studies ont nourri leurs réflexions. Le cercle magique ${ }^{28}$ ou la taxonomie de 
Richard Bartle, répertoriant les différents types de joueurs ${ }^{29}$, ont permis de penser l'immersion à partir du domaine du jeu vidéo, des interfaces et de l'UX. Ces outils, permettant progressivement de faire disparaître toute frontière entre le réel et la fiction et d'orienter la création vers l'utilisateur - à l'image de la gamification-, nécessitent cependant d'être interrogés dans leurs fondements politiques et sociaux, en lien avec les ambitions, objectifs et orientations des créateurs et créatrices.

\section{Le processus créatif face aux dérives de la gamification}

13 Adam Alston, dans son ouvrage Beyond Immersive Theatre: Aesthetics, Politics and Productive Participation ${ }^{30}$, analyse la manière dont le théâtre immersif - et notamment les créations de Punchdrunk - repose sur une participation du public qu'il qualifie de participation entrepreneuriale, s'appuyant sur des valeurs propres à un système néolibéral : «la valorisation du risque, de l'action et de la responsabilité ${ }^{31}$ ». Ce type de pièces s'inscrit dans une logique de récompense fondée sur la participation du public: les spectateurs dans une attitude d'exploration et de prise d'initiative vont être valorisés et vont accéder à des pans de l'œuvre inaccessibles aux moins entreprenants $^{32}$ : «Le citoyen-participant idéal selon cet ethos est l'entrepreneur: l'initiateur, le sujet libre, autonome, motivé, capable, indépendant et consciencieux ${ }^{33}$." Cette critique écrite par Adam Alston trouve un écho particulier dans les jeux vidéo, dont le gameplay fonctionne bien souvent sur un système de motivation et récompense illustré par la boucle " objectif - challenge - récompense ${ }^{34}$ ». L'analogie ne s'arrête pas là, puisque l'auteur pointe également la création par des spectateurs de guides en ligne dictant la meilleure manière de suivre la pièce, ce qui rappelle les sites de solutions de jeux vidéo dévoilant les niveaux, combinaisons de touches et énigmes permettant de réaliser la meilleure performance possible. L'auteur nomme alors le théâtre immersif "the theatre of experience», où l'expérience repose principalement sur une sollicitation sensorielle du participant et où la cohérence de la pièce dépend de son implication. Il devient le héros d'un univers où son action est demandée, valorisée et récompensée.

Les expériences menées par Punchdrunk Enrichment nuancent ces questions, puisqu'elles mettent en avant le collectif, en plus de l'action concrète des participants :

Ce «sentiment de faire partie de quelque chose», le fait d'être tous sur un pied

d'égalité et d'avoir un espace où sa voix (en tant que participant) comptait a aidé à

créer un groupe et une communauté ${ }^{35}$.

14 Il est néanmoins intéressant de confronter ces œuvres aux analyses d'Adam Alston sur la compagnie Punchdrunk. En créant un univers fictif englobant les résidents de manière viscérale, dans le but de les sortir du quotidien de la maison, Punchdrunk propose une expérience proche du parallèle établi par Adam Alston entre l'immersif et le théâtre futuriste de Filippo Tommaso Marinetti : «exciter son public, lui faire oublier la monotonie de la vie quotidienne en le lançant à travers un labyrinthe de sensations ${ }^{36}$ ». La pièce est centrée sur le seul plaisir d'une participation gratuite, dénuée de toute interrogation politique, malgré son inscription au sein d'un ehpad et sa visée sociale et caritative.

15 Toutefois, Punchdrunk prône bel et bien cette participation empreinte de valeurs libérales et entrepreneuriales jusque dans son processus créatif, puisqu'au-delà de la mise en place de résidences, comme cela peut être le cas avec Greenhive Green, la compagnie repose en partie sur un système de bénévolat, avec la participation 
d'artistes en phase de professionnalisation. Adam Alston analyse également le système de financement de la compagnie, fondé sur un accès à des récompenses et exclusivités, comme un écho à cette politique de participation inégale: en supportant financièrement Punchdrunk, ceux que la compagnie nomme les «keyholders ${ }^{37}$ » ont accès à des événements, des cadeaux et des avant-premières inédits.

Cette logique est également présente dans le domaine du jeu vidéo : d'une part par la présence de nombreux projets indépendants sur les plateformes de financement participatif, mais également par la mise en place d'accès anticipés durant les développements. Avant la sortie officielle d'un jeu créé sur ce modèle, les joueurs peuvent acheter le jeu et faire des retours concrets et directs aux développeurs. Ces retours visent souvent à corriger des bugs, des dysfonctionnements, mais peuvent aussi mener à de profondes modifications du système de jeu. La sortie d'un jeu en alpha ${ }^{38}$ ou en bêta ${ }^{39}$ est donc un moyen pour les studios de financer le développement en dehors d'un contrat avec un éditeur, dans une logique participative. Les joueurs, en achetant ces versions en accès anticipé, soutiennent financièrement la création en cours de développement. Ce modèle est surtout utilisé dans le secteur indépendant, mais est également en vogue dans la création de certains titres AAA $^{40}$, à l'image par exemple du jeu Rainbow Six : Quarantine $e^{41}$ du studio Ubisoft.

17 L'ensemble de ces procédés, visant à orienter la création vers une participation active du public - tant dans l'esthétique de l'œuvre que dans le processus créatif-, interrogent sur la place de l'auteur et la portée expressive, politique et artistique de systèmes orientés vers la médiation, la liberté d'action et l'expression du public, où le joueur est productif et producteur. Or, en convoquant un théâtre politique favorisant l'implication du public, le théâtre-forum issu du Théâtre de l'opprimé ${ }^{42}$ d'Augusto Boal, il est possible de dégager des pistes permettant de penser un processus de création communautaire et participatif où le ludique et l'immersif ne reposent plus seulement sur des logiques propres au divertissement et à la gamification, mais sont plutôt des étapes vers une expérimentation artistique, humaine et politique. N'échappant pas à une récupération néolibérale sous couvert de préoccupations humaines et sociales - le théâtre-forum trouve aujourd'hui sa place en entreprise -, le Théâtre de l'opprimé s'inscrit dans un horizon de lutte contre toute forme de domination et d'inégalité.

\section{L'interactivité comme levier émancipateur}

\section{Le théâtre comme libération et émancipation collective différenciée}

Le théâtre-forum occupe encore aujourd'hui une place importante au sein de compagnies de théâtre plaçant l'émancipation et l'expérimentation au cœur de leur ligne artistique. Ce théâtre, développé dans la lignée du théâtre épique de Bertolt Brecht, souhaite affirmer une pratique artistique assise sur une démarche de documentation et de recherche-action.

18 Augusto Boal développe les méthodes du Théâtre de l'opprimé dans les années 1960 et 1970, au Brésil puis en France. Ce théâtre de lutte - défini par Boal lui-même comme une "répétition de la révolution ${ }^{43}$ » et une «arme de libération » - vise à redonner la parole aux opprimés en utilisant le jeu comme pratique libératrice et émancipatrice. Le théâtre-forum, l'une des pratiques développées par Augusto Boal dans ce cadre, a pour objectif de libérer le spectateur d'une oppression et de l'amener à une action directe : 
La poétique de l'opprimé est d'abord celle d'une libération: le spectateur ne délègue aucun pouvoir pour qu'on agisse ou pense à sa place. Il se libère, agit et pense pour lui-même. Le théâtre est action ${ }^{44}$.

19 Une session de théâtre-forum est toujours destinée à un groupe de personnes précis, afin d'agir directement sur les oppressions vécues par le groupe. Une courte scène présentant une injustice sociale est créée grâce à des techniques d'improvisation par une partie du groupe, sous les indications d'un animateur défini au sein du Théâtre de l'opprimé comme le joker. Cette scène - la maquette - est ensuite jouée devant le reste du groupe et chaque participant est invité à proposer des alternatives à la situation en remplaçant un des acteurs : ainsi, la scène évolue de proposition en proposition, vers des pistes de nouveaux rapports de pouvoir et de réflexion sur les problématiques sociales de chaque individu en jeu. Le but est d'expérimenter directement sur scène une multiplicité de points de vue, de cristalliser et mettre en lumière les rapports de domination vécus par le groupe à travers le jeu et de faire naître le débat et une réflexion collective.

20 Augusto Boal insiste sur l'importance donnée au processus dans sa démarche : dans son théâtre, pas de représentation, de spectacle et d'œuvre finie, c'est l'expérimentation et le changement qui définissent sa pratique : «Je crois qu'il est plus important d'arriver à un bon débat qu'à une bonne solution ${ }^{45} \%$. Ce théâtre se situe à l'encontre de ce que le dramaturge nomme le "système tragique coercitif » d'Aristote, mis en place à travers la mimêsis et la catharsis et au sein duquel le spectateur délègue tout pouvoir d'action et est passif : «Il nous faut inventer un mot qui soit l'exact antonyme de catharsis ${ }^{46}$ ».

21 Les conceptrices de The Lost Generation, tout en se reposant sur certains mécanismes et procédés du théâtre immersif - sortie du quotidien, engagement au sein d'un univers englobant le spectateur - organisent un discours critique, conscientes des fondements néo-libéraux de toute pratique participative, et rejoignent in fine les objectifs émancipateurs du théâtre-forum. En se basant sur le mouvement des queer games ${ }^{47}$ et notamment sur les écrits de la théoricienne Bonnie Ruberg, les conceptrices rejettent un système de récompenses valorisant l'attitude entreprenante des participants et vont plutôt encourager la relation avec les acteurs et l'engagement dans la narration d'un point de vue émotionnel, et non sensationnel et viscéral. L'objectif est de sortir d'un schéma qui vise à héroïser le participant. Sa participation n'est plus productive, individuelle et nécessaire ; la pièce va plutôt favoriser la discussion et le dialogue entre les participants et les acteurs par l'improvisation et le jeu de rôle, autour des thématiques engagées de l'œuvre qui aborde les questions sociales et politiques liées à la Génération perdue et à la période des années folles ${ }^{48}$.

Les documents permettant de détailler les différents embranchements narratifs réalisés par Michael Freudenthal sont proches des documents de narrative et game design présents dans l'industrie du jeu vidéo et répondent à une logique algorithmique, schématique et structurelle. À la manière du théâtre-forum, où aucune distinction n'est faite entre acteur et spectateur, l'ensemble du système interactif est pensé dans un souci d'inclusion et d'égalité entre acteurs et participants : à titre d'exemple, il est possible de choisir un « degré d'interaction ${ }^{49}$ » à l'achat du billet, rappelant par certains aspects les paramètres de difficulté des jeux vidéo. Les spectateurs à l'entrée se voient alors attribuer une fiche de personnage - au même titre que les acteurs -, mais également une plume de la couleur correspondant au degré choisi, ce qui permet d'identifier les personnes les plus enclines à un échange. Le système, établi sur des conversations entre les participants, ne valorise pas l'un ou l'autre modèle et aucun 
participant n'est favorisé dans son accès à la narration. Il désamorce la logique de récompense propre au théâtre immersif, où toute implication dans la pièce est attendue et récompensée, et donne la possibilité de se mêler aux acteurs et d'improviser avec eux. Il est également intéressant de noter que le casting de The Lost Generation mêle des acteurs professionnels, mais également des artistes et des amateurs.

\section{Les « play my oppression » de Gonzalo Frasca}

Prouvant encore une fois la richesse des croisements entre jeu vidéo et théâtre, Gonzalo Frasca, concepteur et théoricien, s'appuie sur le théâtre-forum afin de penser un jeu vidéo renouant avec l'humain et la participation du point de vue de l'émancipation. Au-delà d'une logique de marketing plaçant le joueur comme une cible à atteindre, sa proposition envisage le jeu vidéo comme un espace de parole, de témoignage et de remise en question active pour le joueur. Dans sa thèse « Videogames of the oppressed: videogames as a means for critical thinking and debate ${ }^{50} »$ datant de 2001, il repense le cadre d'analyse du jeu vidéo à partir des différentes catégorisations du jeu de Roger Caillois ${ }^{51}$, sur lesquelles s'appuient de nombreuses théories au sein des game studies. Le théâtre-forum devient un cadre pour étudier les spécificités du jeu vidéo comme un espace de simulation permettant d'expérimenter, de «jouer » avec le système dans un monde "bac à sable", afin de le remettre en question: "Les simulations sont des laboratoires pour l'expérimentation où l'action de l'utilisateur n'est pas seulement autorisée mais aussi requise ${ }^{52}$. ». Son jeu September 12th: A Toy World ${ }^{53}$ engage par exemple le joueur à construire un jugement sur le monde dans un processus artistique et expressif, comme analysé par Emmanuelle Jacques : «il inscrit le numérique en tant que médiation des rapports de domination, il pose la question de la réappropriation des moyens d'expression à des fins d'expression citoyenne ${ }^{54}$. »

Le jeu vidéo représente alors un espace contenant les possibilités d'une émergence d'alternatives critiques et citoyennes: "Gonzalo Frasca affirme implicitement que le numérique est un nouveau lieu d'expression possible d'un collectif où peut s'effectuer une transformation en communauté5. espace d'expérimentation active, hors des logiques industrielles propres au médium vidéoludique. Les "play my oppression ${ }^{56}$ ", concept de jeu exposé dans la thèse de Gonzalo Frasca, s'appuient directement sur le fonctionnement d'une séance de théâtreforum : un participant crée un système de jeu fondé sur un modèle existant afin de simuler une situation d'oppression et le poste sur un forum dédié sur internet. Chaque participant peut alors commenter, télécharger le code du prototype et le modifier afin de proposer une variante à la situation. Les mécaniques de jeu y constituent la maquette de théatre-forum et le code informatique devient une matière que chacun est amené à changer afin de proposer différentes alternatives et visions du monde :

Le but des op-games n'est pas d'être capable de représenter une solution concrète au problème d'un participant, mais plutôt de le simuler (généralement métaphoriquement ou métonymiquement) et de l'utiliser comme un objet de réflexion et de discussion ${ }^{57}$.

\section{La participation sous le signe de l'expérimentation critique}

Gonzalo Frasca, en théorisant les play my oppression, mais également en analysant le jeu vidéo du point de vue de la simulation et non de la représentation ${ }^{58}$, appelle à 
considérer le jeu vidéo comme un médium amenant une confrontation entre la vision du monde de l'auteur et l'expression de l'utilisateur, jouant et composant avec les règles du jeu; ce que Mathieu Triclot nomme une "expérience instrumentée " conduisant à un «état ludique $"^{59}$. Il place ainsi la participation des joueurs au sein du système de jeu sous le signe de l'expérimentation critique et de la distanciation recherchée par Bertolt Brecht et Augusto Boal. Aujourd'hui, dans les sphères du jeu vidéo indépendant et amateur, des propositions tentent ainsi d'affirmer la capacité critique et expressive de ce médium, face à une industrie du divertissement où les attentes du marché influent souvent sur les décisions prises en cours de production.

Le mouvement des queer games, qui a directement influencé les créatrices de The Lost Generation dans leur remise en question des procédés liés à la gamification repris par le théâtre immersif, participe à la manière des propositions de Gonzalo Frasca à faire du jeu vidéo un médium de questionnement. Les concepteurs de ces jeux appellent à diversifier les représentations et valeurs véhiculées par le jeu vidéo, mais également à repenser la manière dont il est produit, construit et instrumentalisé. À titre d'exemple, Anna Anthropy ${ }^{60}$, théoricienne et conceptrice de jeux vidéo, promeut une pratique amatrice s'inspirant du mouvement do it yourself (faites-le vous-même), afin d'amener la conception de jeu à davantage d'inclusion. Dans son ouvrage Rise of the videogame zinesters: how freaks, normals, amateurs, artists, dreamers, dropouts, queers, housewives, and people like you are taking back an art form ${ }^{61}$, elle présente un ensemble d'outils et moteurs accessibles permettant à chacun de se saisir du jeu vidéo comme d'un médium artistique.

Ces propositions, issues du champ de l'Artgame ${ }^{62}$ et des jeux brechtiens ${ }^{63}$, invitent alors à repenser la manière dont le jeu vidéo est conçu. À l'encontre des logiques industrielles, où le jeu vidéo doit être rentable et s'inscrire dans un courant dominant, elles nous amènent à considérer le jeu vidéo comme un médium artistique, exprimant des thématiques engagées, critiques et sociales. En laissant une part importante à l'itération, à l'expérience et à l'improvisation, elles font des développeurs des artistes au sein d'ateliers. À la lumière de ce raisonnement, la résidence artistique, qui valorise l'expérimentation, l'inscription dans une communauté et la rencontre avec le public au cours du processus créatif, paraît être une piste pour légitimer le jeu vidéo en tant que pratique artistique issue d'une recherche, d'une documentation et d'un héritage. Les retours des joueurs, au lieu de conduire à une uniformisation de productions souhaitant toucher le plus large public possible, pourraient être un moyen de confronter les valeurs et la vision du monde véhiculées par une création à différents publics afin de faire émerger un débat critique, à la manière du théâtre-forum. Les résidences artistiques s'ouvrent progressivement au jeu vidéo, souvent comme souscatégorie des arts numériques et technologies immersives (réalité virtuelle, réalité augmentée, etc.). Il paraît nécessaire d'affirmer ces pistes permettant de rencontrer un public dans une perspective critique et expérimentale, mais également de considérer le jeu vidéo comme médium ayant pleinement sa place dans les circuits artistiques laissant une place à la recherche, à la prise de risque et à l'itération.

\section{Conclusion}

Le jeu vidéo, élaboré en relation étroite avec le public, repose sur des procédés et ambitions semblables à ceux d'un certain théâtre; les liens entre les deux arts 
s'entremêlent particulièrement autour de la question de l'«immersion ». La volonté de créer un univers cohérent, impliquant pleinement le spectateur ou le joueur et l'embarquant dans la fiction sans résistance, trouve une réponse aujourd'hui dans des technologies de plus en plus performantes, mais également dans des mises en scène pensées à 360 degrés. Bien que les auteurs de théâtre immersif n'assument pas toujours sa part ludique ${ }^{64}$, difficile de ne pas identifier dans ces œuvres une réponse à un glissement de la société vers un besoin de sollicitation constante des individus et d'œuvres culturelles dominées par les attentes et l'expérience des utilisateurs.

Au sein de ces œuvres - tant dans le domaine du jeu vidéo que du théâtre immersif -, des propositions s'affirment cependant comme résistance en interrogeant et en se réappropriant l'ensemble de ces procédés. Dans la lignée d'un théâtre politique Augusto Boal et Bertolt Brecht - et d'un jeu vidéo expérimental et expressif Gonzalo Frasca, les queer games et l'Artgame ${ }^{65}$-, des auteurs s'emparent de ces outils pour amener les joueurs et spectateurs vers une réflexion critique et sociale. La " résidence »-paradigme d'un ensemble de pratiques permettant d'intégrer le joueur et spectateur pleinement au processus créatif - permet alors d'affirmer et construire une pratique artistique consciente d'un contexte socio-culturel, une œuvre collective et émancipatrice, où le processus de création n'est plus seulement étape préparatoire, mais devient pleinement expérience esthétique.

La résidence menée à la villa Kujoyama au Japon en 2019 par le développeur Christophe Galati, premier créateur de jeux envoyé en résidence artistique et soutenu financièrement par l'Institut français ${ }^{66}$, est un exemple de la manière dont le jeu vidéo peut côtoyer d'autres pratiques artistiques et s'inspirer d'un contexte socioculturel, afin de s'établir dans une réelle démarche de recherche, de documentation et de réflexion. La résidence permet ici de rencontrer un ensemble d'artistes mais aussi un public - dans le cadre de la résidence, Christophe Galati est amené à exposer les documents de game et narrative design ${ }^{67}$, les ressources graphiques mais également le code de son jeu Projet Himitsu - et de légitimer le jeu en tant qu'œuvre d'art témoin d'une époque et d'un lieu.

\section{BIBLIOGRAPHIE}

Alston Adam, « Audience participation and neoliberal value : risk, agency and responsibility in immersive theatre ", Performance Research [en ligne], vol. 18, n 2, p. 128-138, 2013.

Alston Adam, Beyond Immersive Theatre: Aesthetics, Politics and Productive Participation, Basingstoke, Palgrave Macmillan, 2016.

Anthropy Anna, Rise of the videogame zinesters: how freaks, normals, amateurs, artists, dreamers, dropouts, queers, housewives, and people like you are taking back an art form, New York, Seven Stories Press, 2012. 
Begault Marine, Hodson Kate et Langford Susan, Magic Me's artists residencies in care homes [en ligne], Londres, Allen \& Overy, 2017. URL : https://magicme.co.uk/resource/artist-residenciesin-care-homes-report/ [consulté le 17/02/2021]

Boal Augusto, Jeux pour acteurs et non-acteurs. Pratique du Théâtre de l'opprimé, trad. du portugais V. Rigot-Müller, Paris, La Découverte, 2004.

Boal Augusto, Théâtre de l'opprimé [1975], trad. du portugais D. Lémann, Paris, La Découverte, 2007.

Caillois Roger, Les Jeux et les Hommes (le masque et le vertige) [1958], Paris, Gallimard, coll. « Folio. Essais », 1992.

Cros Edmond, La Sociocritique, Paris, L'Harmattan, coll. « Pour comprendre », 2003.

Deterding Sebastian et al., « From game design elements to gamefulness: defining "gamification" ", in Proceedings of the 15th International Academic MindTrek Conference: Envisioning Future Media Environments [en ligne], Tampere, 28-30 septembre, New York, Association for Computing Machinery, 2011, p. 9-15.

Frasca Gonzalo, «Simulation versus narrative: introduction to ludology », in Mark Wolf et Bernard Perron (dir.), The Video Game Theory Reader, New York, Routledge, 2003, p. 221-236.

Frasca Gonzalo, «Videogames of the oppressed: videogames as a means for critical thinking and debate ", mémoire de master 2 en design et technologie de l'information, sous la direction de Janet H. Murray, Institut de technologie de Géorgie, 2001.

Huizinga Johan, Homo ludens. Essai sur la fonction sociale du jeu [1938], Paris, Gallimard, coll. « Tel », 1988.

Jacques Emmanuelle, « Des jeux vidéo engagés, pour éduquer ? Du théâtre de l'opprimé aux jeux vidéo : September 12th de Gonzalo Frasca », Rivista dell'Istituto di Storia dell'Europa Mediterranea, vol. 2, $\mathrm{n}^{\circ} \mathrm{II}, 2018$, p. 65-76.

Jenkins Henry, "Game design as narrative architecture », in Noah Wardrip-Fruin et Pat Harrigan (dir.), First Person. New Media as Story, Performance, and Game, Cambridge, The MIT Press, 2004, p. 118-130.

Laurel Brenda, Computers as Theatre [1991], 2 éd., Reading, Addison-Wesley, 2014.

Machon Josephine, Immersive theatres: intimacy and immediacy in contemporary performance, Basingstoke, Palgrave Macmillan, 2013.

Salen Katie et Zimmerman Eric, Rules of Play. Game Design Fundamentals, Cambridge, The MIT Press, 2003.

Schrank Brian, Avant-garde videogames: playing with technoculture, avant-propos D. Bolter, Cambridge, The MIT Press, 2014.

Siegel Claire, «L'Artgame, un jeu utopique à l'ère de la gamification? ", thèse de doctorat en arts plastiques, sous la direction de Valérie Arrault, Montpellier 3, 2015.

Siegel Claire, « Les origines socio-idéologiques de l'Artgame. Enjeux de la gamification dans la création vidéoludique ", in Patricia Signorile (dir.), Les cahiers des rencontres Droit \& Arts. La création numérique ludique : une cuvre comme les autres ?, Aix-en-Provence, Presses universitaires d'AixMarseille, 2019, p. 31-48.

Siegel Claire, « Valeurs et représentations au cœur du processus de gamification. Approche sociocritique des symptômes d'une société du jeu total par trois de ses formes : serious games, 
Games for Change et artgames ", Interfaces numériques [en ligne], vol. 3, $\mathrm{n}^{\circ}$ 3, 2014, p. 497-520.

URL : https://www.unilim.fr/interfaces-numeriques/1594 [consulté le 17/02/2021]

Stenros Jaakko, « In defence of a magic circle: the social, mental and cultural boundaries of

play ", Transactions of the Digital Games Research Association, vol. 1, n 2, 2014.

Triclot Mathieu, Philosophie des jeux vidéo, Paris, La Découverte, coll. « Zones », 2011.

\section{Pièces de théâtre}

Punchdrunk, Greenhive Green, présenté à la maison de soins Greenhive, Londres, 2016.

Madame Lupin, The Lost Generation, présenté aux Belles Poules, Paris, 2019.

\section{NOTES}

1. Brenda Laurel, Computers as Theatre [1991], Reading, Addison-Wesley, 2014.

2. Josephine Machon, Immersive theatres: intimacy and immediacy in contemporary performance, Basingstoke, Palgrave Macmillan, 2013.

3. https://magicme.co.uk/

4. https://www.anchorhanover.org.uk/

5. Je traduis « The four arts partners were all prepared to take on the challenge of making work with, and for, this very different audience, in a very particular environment, whilst keeping the integrity of their own practice and its unique aesthetic. ", Marine Begault, Kate Hodson et Susan Langford, Magic Me's artists residencies in care homes [en ligne], Londres, Allen \& Overy, 2017. URL : https://magicme.co.uk/resource/artist-residencies-in-care-homes-report/ [consulté le $17 / 02 / 2021]$

6. Le compte Twitter @GreenhiveGreen et une lettre d'information suivant les péripéties du village relatent ces prises de décision et les événements du village. À titre d'exemple, le comité va, par exemple, pouvoir choisir l'hymne des villages lors d'un concert organisé à l'Ehpad, décorer des tasses, écrire des poèmes, mais aussi interagir avec le village voisin de manière épistolaire.

7. Je traduis «[...] space is totally transformed visually, with atmospheric soundtracks, smells, textures and lighting. The ambition was to create a beautiful space for residents that would enhance their environment and provide a point of interest in the home, but also transport residents to a different environment that was part of our story world. ", ibid.

8. Je traduis « We began describing the project as part game and part soap-opera », ibid.

9. Je traduis «The planned narrative about the history of the two villages was made simpler shortly after we began the 8 week residency, as we realised that it was unnecessary and hard for many residents to retain from week to week. The story became about two neighbouring villages becoming friends through exchanges - from sending Diplomacy Cakes, to choosing a village anthem. ", ibid.

10. L'une des caractéristiques de la compagnie est de proposer aux spectateurs une déambulation au sein du lieu de représentation, souvent très étendu. Les participants portent chacun un masque permettant de les séparer du groupe d'acteurs.

11. Je traduis « At its simplest the project was a nice space which we could meet in each week and share a cup of tea and do a fulfilling activity. On a more complex level it was a place where a narrative was unfolding, a narrative of which they are at the centre and are the protagonists, a world of mystery and detail. », ibid. 
12. Thomas McMullan, "The immersed audience: how theatre is taking its cue from video games ", The Guardian [en ligne], 20 mai 2014. URL : https://www.theguardian.com/technology/ 2014/may/20/how-theatre-is-taking-its-cue-from-video-games

13. Gone Home, Portland, Fullbright, 2013.

14. Punchdrunk, The Drowned Man: A Hollywood Fable, présenté aux Temple Studios, Londres, 2013. 15. Henry Jenkins, "Game design as narrative architecture", in Noah Wardrip-Fruin et Pat Harrigan (dir.), First Person. New Media as Story, Performance, and Game, Cambridge, The MIT Press, 2004, p. 118-130.

16. La gamification, comme définie par Sebastian Deterding dans son «From game design elements to gamefulness: defining "gamification" » en 2011, désigne l'utilisation du jeu et de ses mécanismes dans des contextes de non-jeu. Le jeu est utilisé comme levier de performance et de productivité, afin de motiver et d'engager les individus à l'aide de mécanismes ludiques dans des domaines comme le travail, l'éducation ou le sport.

17. Claire Siegel, "L'Artgame, un jeu utopique à l'ère de la gamification ? ", thèse de doctorat en arts plastiques, sous la direction de Valérie Arrault, Montpellier 3, 2015, p. 39.

18. Gordon Calleja, In-game : from immersion to incorporation, Cambridge, The MIT Press, 2011.

19. Le terme gameplay, parfois traduit en français par "jouabilité », désigne la relation entre le système de jeu, ses règles, et l'attitude ludique qu'il initie chez les joueurs et joueuses.

20. entretien avec Mélanie Dorey et Michael Freudenthal réalisé par Olivia Levet le 7 mai 2020

21. À la demande de Michael Freudenthal et Mélanie Dorey, leur binôme sera considéré comme féminin tout au long de cet article.

22. « immersive party ", ibid.

23. Michael Freudenthal a travaillé au CRI - Center of research and interdisciplinarity, université de Paris - et étudie actuellement les sciences du jeu à l'université Sorbonne Paris Nord.

24. La dénomination de "play designer", venant remplacer celle, plus commune, de "game designer ", affirme une conception par le prisme de l'attitude ludique. Au sein des games studies et sciences du jeu, la distinction anglaise entre les termes "game», le jeu du point de vue de son système de règles, et « play », le jeu du point de vue de sa réception par les joueurs, est porteuse de sens.

25. Michael Freudenthal, ibid.

26. "sans couture ». Le terme "seamless» est issu du domaine de l'informatique ubiquitaire conceptualisé par Mark D. Weiser et renvoie à une communication invisible et transparente entre différents systèmes connectés.

27. « intégration ». Le terme est issu du domaine de l'UX visant à penser, théoriser et fluidifier les différentes étapes de prise en main d'une interface par l'utilisateur.

28. Johan Huizinga, Homo ludens. Essai sur la fonction sociale du jeu [1938], Paris, Gallimard, coll. «Tel», 1988; Katie Salen et Eric Zimmerman, Rules of Play. Game Design Fundamentals, Cambridge, The MIT Press, 2003 ; Jaakko Stenros, « In defence of a magic circle: the social, mental and cultural boundaries of play », Transactions of the Digital Games Research Association, vol. 1, $\mathrm{n}^{\circ} 2$, 2014.

29. Richard Bartle, « Hearts, clubs, diamonds, spades: players who suit MUDs » [en ligne], Journal of MUD research, vol. 1, no 1, 1996. URL: https://www.researchgate.net/publication/ 247190693_Hearts_clubs_diamonds_spades_Players_who_suit_MUDs [consulté le 17/02/2021]

30. Adam Alston, Beyond Immersive Theatre: Aesthetics, Politics and Productive Participation, Basingstoke, Palgrave Macmillan, 2016.

31. Je traduis "the valorisation of risk, agency and responsibility. ", Alston Adam, "Audience participation and neoliberal value: risk, agency and responsibility in immersive theatre", Performance Research [en ligne], vol. 18, nº 2, p. 128-138, 2013. 
32. Les " one-on-one" sont l'une des caractéristiques principales des pièces de la compagnie Punchdrunk : il s'agit de moments privilégiés seul à seul entre un acteur et un participant choisi dans le public.

33. Je traduis " The ideal citizen-participant according to this ethos is the entrepreneur: the selfstarter, the independent, autonomous, motivated subject who is capable, self-reliant and conscientious ", Alston Adam, Beyond Immersive Theatre: Aesthetics, Politics and Productive Participation, op. cit., p. 130.

34. Cette boucle correspond à la boucle de gameplay définie par Marc Albinet dans son ouvrage Concevoir un jeu vidéo (Limoges, Fyp, coll. «Entreprendre », 2010), présente dans la plupart des productions vidéoludiques.

35. Je traduis « This 'feeling part of something', being on an equal footing and having a space where your voice (as a participant) was important helped to build group and community.", Marine Begault, Kate Hodson et Susan Langford, op. cit.

36. Je traduis « excite its audience, that is, make it forget the monotony of daily life, by sweeping it through a labyrinth of sensations ", citation de Fillipo Tommaso Marinetti issue du Manifeste du futurisme, in Adam Alston, Beyond Immersive Theatre: Aesthetics, Politics and Productive Participation, op. cit., p. 110.

37. https://www.punchdrunk.org.uk/support-us/

38. première version d'un jeu vidéo, ne présentant qu'une partie de l'œuvre finale

39. version plus avancée du développement, présentant l'ensemble des fonctionnalités du jeu et visant surtout à la correction de bugs et dysfonctionnements du système

40. jeux vidéo pour lesquels les studios disposent de budgets très importants

41. Ubisoft Montréal, Tom Clancy's Rainbow Six: Quarantine, Rennes, Ubisoft, initialement prévu pour 2020.

42. Augusto Boal, Théâtre de l'opprimé [1975], Paris, La Découverte, 2007.

43. Ibid., p. 48.

44. Id.

45. Augusto Boal, Jeux pour acteurs et non-acteurs. Pratique du Théâtre de l'opprimé, Paris, La Découverte, 2004, p. 262.

46. Ibid., p. 26.

47. Les queer games sont des jeux créés par des concepteurs et conceptrices se définissant comme queer et visant à diversifier les représentations standardisées et stéréotypées des jeux vidéo, en se réappropriant le médium en tant que médium d'expression.

48. Les conceptrices se sont par ailleurs appuyées sur des outils issus du jeu de rôle - notamment le livret «Les années folles » à destination des joueurs du jeu de rôle L'Appel de Cthulhu - pour établir des fiches de personnage à destination des acteurs et documenter l'époque.

49. Furtif ("vous choisissez d'assister discrètement à l'histoire »), jaseur ("vous choisissez de faire partie de l'histoire sans être au centre de l'attention ») et hardi (« vous choisissez d'avoir un impact sur l'histoire et d'être plus proche des personnages »), site officiel de The Lost Generation, [en ligne]. URL: https://web.archive.org/web/20200806221821/thelostgeneration.fr/lexperience [consulté le 17/02/2021]

Ce choix ne limite pas leur expérience, il sera seulement un signe visible - incarné par une plume de couleur portée à la boutonnière - indiquant aux actrices et acteurs à quel point encourager leur implication.

50. Gonzalo Frasca, «Videogames of the oppressed: videogames as a means for critical thinking and debate ", mémoire de master 2 en design et technologie de l'information, sous la direction de Janet H. Murray, Institut de technologie de Géorgie, 2001.

51. Roger Caillois, Les Jeux et les Hommes (le masque et le vertige) [1958], Paris, Gallimard, coll. « Folio. Essais », 1992. 
52. Je traduis «Simulations are laboratories for experimentation where user action is not only allowed but also required.", Gonzalo Frasca, "Simulation versus narrative: introduction to ludology ", in Mark Wolf et Bernard Perron (dir.), The Video Game Theory Reader, New York, Routledge, 2003, p. 221-236.

53. Gonzalo Frasca, September 12th: A Toy World, 2010.

54. Emmanuelle Jaques, « Des jeux vidéo engagés, pour éduquer? Du théâtre de l'opprimé aux jeux vidéo: September 12th de Gonzalo Frasca », Rivista dell'Istituto di Storia dell'Europa Mediterranea, vol. 2, nº II, 2018, p. 65-76.

55. Id.

56. « joue mon oppression"

57. Je traduis «the goal of op-games is not to be able to represent a concrete solution to the participant's problem, but rather to simulate (usually metaphorically or metonymically) it and use it as an object to think and discuss with ", Gonzalo Frasca, «Videogames of the oppressed: videogames as a means for critical thinking and debate ", mémoire de master 2 en design et technologie de l'information, sous la direction de Janet H. Murray, Institut de technologie de Géorgie, 2001, p. 101.

58. L'analyse de Gonzalo Frasca fait écho au débat entre ludologues et narratologues, reflétant la recherche d'un cadre pour analyser le jeu vidéo au-delà de ses capacités narratives.

59. Mathieu Triclot, Philosophie des jeux vidéo, Paris, La Découverte, coll. « Zones », 2011.

60. https://itch.io/profile/w

61. Anna Anthropy, Rise of the videogame zinesters: how freaks, normals, amateurs, artists, dreamers, dropouts, queers, housewives, and people like you are taking back an art form, New York, Seven Stories Press, 2012.

62. Le champ de l'Artgame, tel que le définit Claire Siegel dans son article « Les origines socioidéologiques de l'Artgame, enjeux de la gamification dans la création vidéoludique » en 2020, désigne des jeux vidéo au caractère artistique. Ce mouvement s'est développé à partir des années 2000 aux États-Unis et en Italie et regroupe un ensemble de courants visant à considérer le jeu vidéo comme médium artistique, expressif et critique.

63. Brian Schrank, Avant-garde videogames: playing with technoculture, avant-propos D. Bolter, Cambridge, The MIT Press, 2014.

64. Léonard Matton, metteur en scène et directeur du lieu Le Secret, revendique dans l'émission "Qu'est-ce que le théâtre immersif» un éloignement des pratiques ludiques telles que l'escape game, qui amène une forme de "condescendance " dans l'appréciation de ce type de théâtre. Léonard Matton, «Qu'est-ce que le théâtre immersif », Signes des temps [en ligne], France Culture, 30 décembre 2018. URL: https://www.franceculture.fr/emissions/signes-des-temps/quest-ceque-le-theatre-immersif

65. Dans sa thèse "L'Artgame, un jeu utopique à l'ère de la gamification? ", Claire Siegel interroge les capacités utopiques de l'Artgame. Ce mouvement, en considérant les procédés ludiques dans leur pouvoir émancipateur et critique, appelle à envisager le jeu vidéo comme résistance aux idéologies dominantes et aux valeurs véhiculées par le processus de gamification.

66. Christophe Galati a participé au programme dans la discipline « productions digitales », le jeu vidéo n'ayant pas fait partie du programme de la villa Kujoyama jusque-là. https:// www.villakujoyama.jp/resident/christophe-galati/

67. Le terme narrative design renvoie à la conception de la structure narrative d'un jeu vidéo et à la manière dont elle est transmise aux joueurs et joueuses. 


\section{RÉSUMÉS}

Le jeu vidéo et les interfaces numériques, durant leur développement, se sont nourris du théâtre et des arts vivants, afin d'intégrer la prise en compte de l'utilisateur et de l'humain dans leur conception. Aujourd'hui l'apparition d'un «théâtre immersif» confirme ce rapprochement. À travers l'analyse sociocritique du processus créatif de deux œuvres théâtrales, Greenhive Green de Punchdrunk Enrichment et The Lost Generation de Madame Lupin, cet article analyse de manière croisée la résidence en théâtre et la mise en place de playtests et de versions anticipées dans le domaine du jeu vidéo. Possédant un vocabulaire, des procédés et des ambitions communs, le théâtre immersif et le jeu vidéo semblent constituer un miroir à une idéologie dominante instaurant une ère du participatif, où l'individu entrepreneur, autonome et indépendant est valorisé au détriment des autres, comme le suggère l'analyse d'Adam Alston du travail de la compagnie Punchdrunk. Pourtant, à l'image d'un théâtre participatif politique - le Théâtre de l'opprimé d'Augusto Boal -, certains artistes se réapproprient ces outils afin de construire une œuvre de manière collaborative, critique et à contre-courant, où la rencontre avec un public permet de coconstruire une expérience esthétique tournée vers l'émancipation.

Video games and human-computer interfaces, in their development, were fed by drama and performing arts in order to integrate the consideration of the user and the human in their design. Today, the appearance of an "immersive theater" confirms this rapprochement. Through the sociocritical analysis of the creative process of two plays, Greenhive Green by Punchdrunk Enrichment and The Lost Generation by Madame Lupin, this article draws a parallel between theater residency programs and playtests or early versions in the video game industry. Through a common vocabulary, processes and ambitions, immersive theater and video game seem to constitute a mirror to a dominant participatory ideology where the entrepreneurial, autonomous and self-reliant is valued, as suggested in the Adam Alston's analysis of the Punchdrunk company's work. However, like a participatory political theater - the Theatre of the oppressed developed by Augusto Boal -, some artists are appropriating these tools in order to construct art in a collaborative, critical way and in resistance to industry, where audience integration makes the co-construction of an aesthetic experience turned towards emancipation possible.

\section{INDEX}

Mots-clés : théâtre, jeux vidéo, théâtre-forum, théâtre immersif, prototype, artgame, résidence

\section{AUTEURS}

\section{OLIVIA LEVET}

Doctorante en arts, spécialité arts plastiques, Université Paul-Valéry-Montpellier, Représenter, inventer la réalité, du romantisme au Xxi ${ }^{\mathrm{e}}$ siècle (Rirra 21), 71 rue du Professeur-Henri-Serre 34090 Montpellier ; olivia.levet@univ-montp3.fr 